\title{
Tissue typing with ultrasound RF time series: Phantom studies
}

\author{
Mehdi Moradi ${ }^{a}$, Parvin Mousavi ${ }^{c}$, Robert Rohling ${ }^{a, b}$ and Purang Abolmaesumi ${ }^{c}$ \\ ${ }^{a}$ Department of Electrical and Computer Engineering, University of British Columbia; \\ ${ }^{b}$ Department of Mechanical Engineering, University of British Columbia, \\ Vancouver, BC, CANADA; \\ ${ }^{c}$ School of Computing, Queen's University, Kingston, ON, CANADA. \\ Email: moradi@ece.ubc.ca
}

\begin{abstract}
We report phantom studies on a new approach to ultrasound-based tissue typing. In the proposed approach, we continuously record RF echo signals backscattered from tissue, while the imaging probe and the tissue are fixed in position. The continuously recorded RF data generates a time series of echoes for each spatial sample of the RF signal. We use the spectral and fractal features of ultrasound RF time series averaged over a region of interest, along with support vector machine classifiers, for tissue typing. In this paper, the effects of two properties of tissue on RF time series are investigated: cell size and elasticity. We show that RF time series acquired from agar-gelatin based tissue mimicking phantoms, with difference only in the size of cell-mimicking glass beads, are distinguishable with statistically reliable accuracies up to $82.2 \%$. Similar experiments using phantoms with different elastic properties did not result in consistently high classification accuracies. The results of this study confirm that the evident differences in microstructure of the cancerous versus normal tissue could play a role in the success of the proposed tissue typing method in detection of prostate cancer.
\end{abstract}

Keywords: Ultrasound-based tissue typing, RF time series, prostate cancer, support vector machines, tissuemimicking phantoms.

\section{INTRODUCTION}

Ultrasonic tissue typing methods assume that physical characteristics of human tissue change during the formation of pathological conditions. These changes can result in observable alterations in backscattered ultrasound signals. One group of techniques to quantify the disease-specific patterns in backscattered ultrasound rely on features extracted from the calibrated average spectrum of RF signals. This technique has been applied to diagnosis of cancer lesions in liver, breast and prostate. ${ }^{1}$

In the case of prostate cancer, the most common cancer among men in North America, ${ }^{2}$ ultrasound-based tissue typing has not found widespread clinical usage. Prostate tumors lack visually distinct appearances on ultrasound images. Therefore, pathologically significant cases of cancer can be missed during ultrasoundguided biopsy, resulting in false negative or repeated biopsies. The goal of our research is to augment the ultrasound images with tissue typing information that can enable targeted biopsies. To this end, we have proposed a new approach to extract tissue typing parameters from ultrasound RF signals. Our approach is based on the observation that if a specific location in tissue undergoes continuous interactions with the ultrasound signal, the time series of echoes from that location carries "tissue typing" information. In other words, although variations in the intensity of a spatial sample of RF echo over time are partly due to noise, they depend on the tissue type as well. We have proposed seven tissue typing parameters ${ }^{3}$ extracted from the time series of samples of RF signals and used them to classify regions of prostate tissue of 16 patients in cancerous and normal classes (area under ROC curve of $0.89^{4}$ ). We have also shown that the RF times series features form a very accurate set of parameters to distinguish different types of animal tissues. ${ }^{5}$

Although the tissue typing capabilities of RF time series are evident through our experimental studies, the origin of tissue-type related information in the sequence of ultrasound echoes is not clear. Our current research is partly aimed at identifying the specific physical parameters of tissue that affect RF time series

Medical Imaging 2009: Ultrasonic Imaging and Signal Processing, edited by Stephen A. McAleavey, Jan D'hooge Proc. of SPIE Vol. 7265, 726516 - (C) 2009 SPIE - CCC code: 1605-7422/09/\$18 - doi: 10.1117/12.811693 
Table 1. Dry weight percentage of the various components in the particle size phantoms.

\begin{tabular}{cc} 
Component & Weight \% \\
\hline Agar & 1.17 \\
Gelatin & 3.6 \\
Glass beads & 6 \\
Bleach & 0.25 \\
Water & 88.98 \\
\hline
\end{tabular}

features. To this end, in this paper we report phantom studies on two potentially influential tissue properties: cell size and elasticity. During the formation of prostate cancer, both of these properties are affected.

At the microscopic level, prostate tumors are composed of immature and "small cells" which lack structural organization. ${ }^{6}$ Previous studies have shown that the size and the microstructure of the sub-wavelength particles affect the backscattered signals ${ }^{7}$ and the spectral parameters extracted from single RF frames. ${ }^{1}$ To determine the effects of cell size on RF time series, in the current study we acquired and studied ultrasound RF time series from custom tissue mimicking phantoms with varied microscopic particle sizes.

At the macroscopic level, reasonably large tumors are palpably harder than normal tissue. This fact forms the basis for digital rectal examination and also elastography ${ }^{8}$ which relies on comparison of RF echoes received from tissue before and after applying an external compression. Investigators have previously studied the application of highly focused ultrasound to produce the required compression profile for elastography. ${ }^{9}$ We study the possible utility of RF time series for elastography without applying compression.

In the remainder of the paper, we describe the phantom building process, data collection, and classification using Support Vector Machines (SVM) (Section 2), present the classification results (Section 3), provide some discussions on the possible source of tissue typing information in RF time series (Section 4) followed by conclusions (Section 5).

\section{METHODS}

\subsection{Phantoms}

We built tissue mimicking phantoms in the form of gelatin-agar based suspensions. ${ }^{10,11}$ For elasticity phantoms, higher agar concentrations produced greater stiffness. Cellulose was added to create the tissue-like speckle patterns. For particle size phantoms, microscopic glass beads with known distributions of particle sizes were added instead of cellulose. In all cases, gelatin and agar (Sigma Aldrich, Canada, Oakville, ON) were mixed in distilled water in room temperature and while being constantly stirred, were heated to $90^{\circ} \mathrm{C}$ at which point cellulose (Sigma Aldrich Corp.) or glass beads (Potters Industries, Parsippany, NJ) were added. Minute quantities of bleach were added to avoid bacterial growth in the phantoms. ${ }^{12}$ The resulting mix was slowly cooled down to the room temperature, while being stirred. Upon arrival at the room temperature, the suspension was transferred to a plastic box and stored overnight in a cooler. The ultrasound data was collected the next day.

Particle size phantoms: The dry weight percentages of ingredients used in particle size phantoms are given in Table 1. Two different types of these phantoms were built which shared the same recipe, with the only difference being the size of microscopic glass beads. The two types of utilized glass beads were of average diameter of $35 \mu M$ (Potters Industries catalog number: 3000E used in $P 1$ phantoms) and $11 \mu M$ (Potters Industries catalog number: $5000 \mathrm{E}$ used in $P 2$ phantoms). For comparison, it should be noted that the prostate PC-3 cell line has a mean diameter of $26 \mu M{ }^{7}$ The detailed distribution of bead sizes used in $P 1$ and $P 2$ phantoms materials are provided in Table 2.

Our measurements and supplier data for the materials show that the physical properties of the phantoms (such as density, elasticity, and speed of sound) were similar. This was important since the phantoms were intended to be different only in terms of cell sizes. The glass beads used in $P 1$ and $P 2$ phantoms had very close 
Table 2. The distribution of the bead sizes in glass beads used for the particle size phantoms ( $P 1$ and $P 2$ phantoms).

\begin{tabular}{ccc} 
& $P 1$ & $P 2$ \\
\hline 10\% finer than & $18 \mu m$ & $3 \mu m$ \\
$50 \%$ finer than & $35 \mu m$ & $9 \mu m$ \\
90\% finer than & $65 \mu m$ & $38 \mu m$ \\
Mean value & $35 \mu m$ & $11 \mu m$ \\
\hline
\end{tabular}

P1 Phantoms

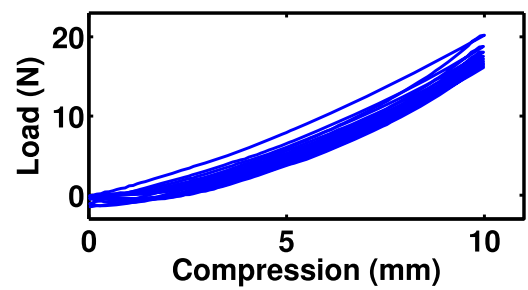

P2 Phantoms

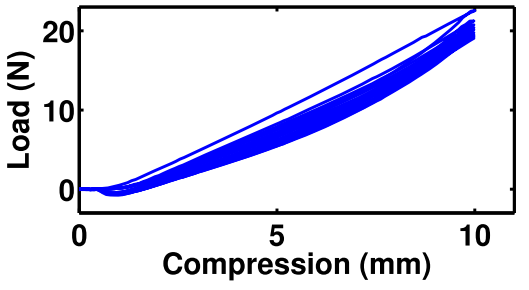

Figure 1. The stress-strain curves for the particle size phantoms.

untapped densities* (101 vs. $99 \mathrm{lbs} / \mathrm{ft}^{3}$ ). The resulting phantoms, also had very similar elastic properties. The stress-strain curves acquired using an Instron 5500R materials test system, are depicted in Figure 1. Since the speed of sound is related to density and stiffness $(c=\sqrt{K / \rho}$ where $K$ is the bulk modulus and $\rho$ is the density $^{13}$ ), the similar values of elasticity and density mean that the speed of sound should also be similar in the two types of phantoms. For cross-validation purposes, two copies of the $P 1$ phantom and two of the $P 2$ phantom were built (four particle size phantoms in total). It should be noted that the B-scan images acquired from the two types of the phantoms appeared very similar (Figure 2).

Elasticity phantoms: We built four different types of elasticity phantoms. The composition of the materials in these phantoms, which we call $E 1, E 2, E 3$ and $E 4$, are given in Table 3 . Based on, ${ }^{10} E 1$ and $E 2$ can respectively be used as the substrate and the hard inclusion of a elastography phantom. $E 3$ and $E 4$ form another suitable pair for elastography. In the current study, we built $E 1, E 2, E 3$ and $E 4$ as four separate phantoms. The stress-strain curves for these phantoms are given in Figure 3. Due to the high concentration of solid suspension components, the process of building homogenous elastography phantoms of types $E 1, E 2$, $E 3$ and $E 4$ had more variability and the stress-strain measurements (Figure 1) show that the final result is not as homogenous as particle size and $E 1$ phantoms. The average slopes of ten measurements of the stressstrain curves for $E 1, E 2, E 3$ and $E 4$ were respectively: $3.3 \pm 0.5,9.5 \pm 1.7,6.6 \pm 1.5$ and $9.9 \pm 1.9 \mathrm{~N} / \mathrm{mm}$. For

${ }^{*}$ Untapped bulk density is the lowest attainable density for a material.

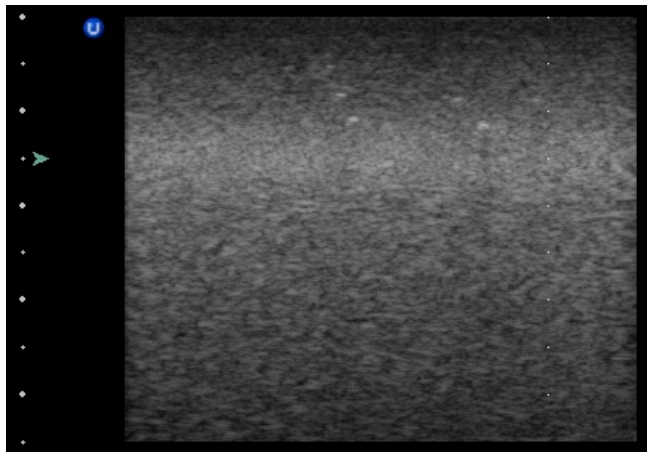

$(P 1)$

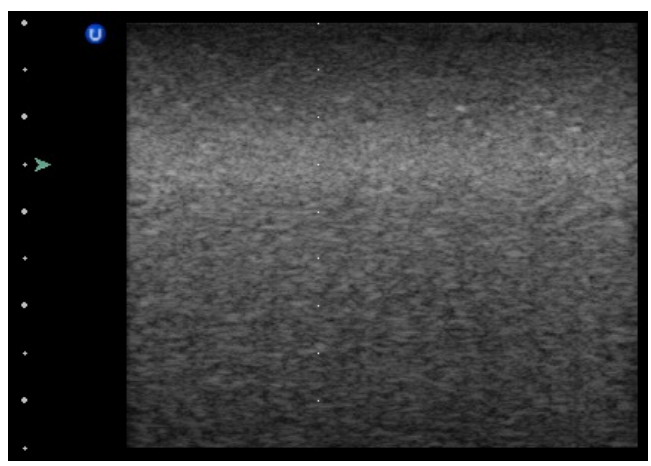

$(P 2)$

Figure 2. The typical B-scan images of $P 1$ and $P 2$ phantoms. The textures appeared to be visually identical. 
Table 3. Dry weight percentage of the various components in the elasticity phantoms.

\begin{tabular}{c|cccccc} 
& Agar & Gelatin & Cellulose & Glycerol & Bleach & Water \\
\hline$E 1$ & 1.17 & 3.6 & 3 & 4 & 0.25 & 87.98 \\
$E 2$ & 3.53 & 3.6 & 3 & 2 & 0.25 & 87.62 \\
$E 3$ & 1.17 & 5.52 & 3 & 2 & 0.25 & 88.06 \\
$E 4$ & 3.64 & 5.52 & 3 & 1 & 0.25 & 86.59 \\
\hline
\end{tabular}
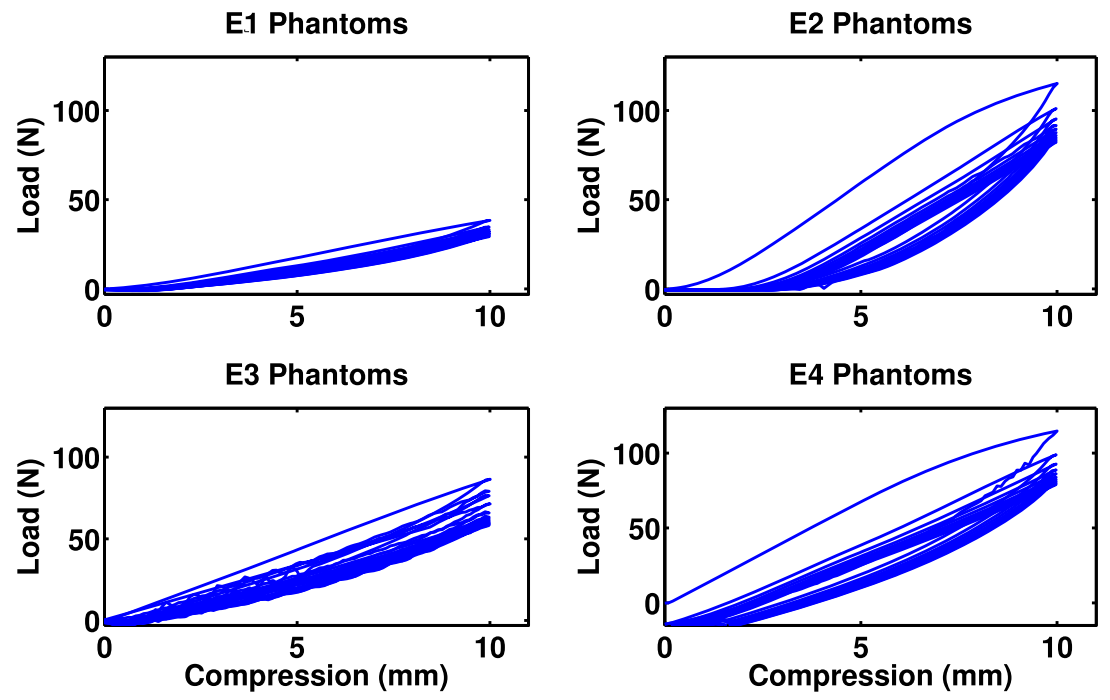

Figure 3. The stress-strain curves for the elasticity phantoms.

cross-validation purposes, two copies of each phantom type were built (eight elasticity phantoms in total). It should be noted that appropriate amounts of glycerol were added to the elasticity phantoms to regulate the ultrasonic propagation speeds to $1540 \mathrm{~m} / \mathrm{s}$ which matches the typical speed of sound in soft tissue. ${ }^{10}$

\subsection{RF time series}

A cylindrical opening was created in the phantoms just before congealing by inserting a plastic pipe into one corner of the holding boxes. Before imaging, this pipe was removed carefully and a trans-rectal ultrasound probe (Model: BPSL9-5/55/10 Vermon, France, center frequency: 6.6 $\mathrm{MHz}$, number of elements: 128, sampling rate of the RF: $20 \mathrm{MHz}$ ) was inserted vertically into the hole left behind and was fixed with a standing clamp (Figure 4). The data was collected using a Sonix RP (Ultrasonix Inc., Richmond, BC, Canada) ultrasound machine. To form the RF time series, we acquired 128 frames of RF data from eight different cross sections within each phantom. Therefore, the length of RF time series in our analysis of phantom data was 128. The data was acquired at 22 frames per second (fps). We also acquired data at 45 and 15 fps to study the effects of frame rate on the performance of RF time series features. A single transmit focal point was set at $1.5 \mathrm{~cm}$ and only data from the focal zone $(1 \mathrm{~cm}$ on each side of the focal point) were used for feature extraction and classification experiments. The RF sampling rate was $20 M H z$ and the number of bits per RF sample was 16 .

\subsection{Tissue typing features extracted from RF time series}

We extracted the RF time series features from areas of interest (ROIs) of size $1 \mathrm{~mm}$ in lateral direction and $3.5 \mathrm{~mm}$ in axial direction. This included four segments of RF lines, each of length 96 samples. Therefore, each ROI was formed by $384 \mathrm{RF}$ time series $(4 \times 96)$. Each time series was of length 128 . 


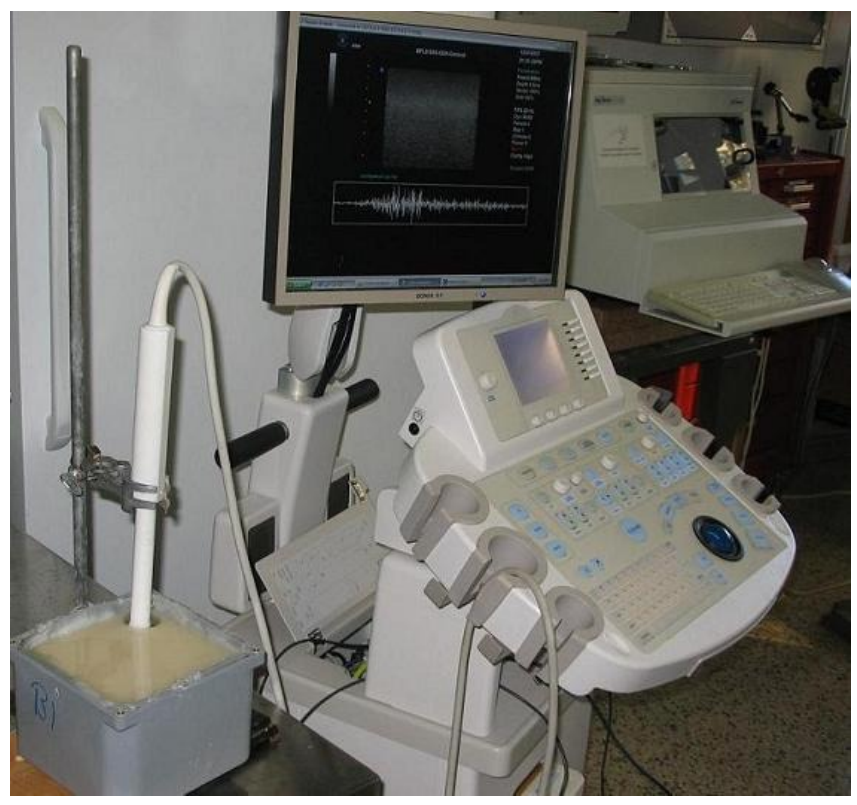

Figure 4. Data collection setup.

A total of 984 ROIs of each phantom type were available which equally originated from the two versions built from each phantom type. Seven features were extracted from the ROIs for tissue typing which were similar to the ones that we proposed in. ${ }^{3}$ Therefore, we only briefly describe the features here.

Spectral features of the RF time series (S1-S6): Each RF time series was a discrete signal of length 128 in our dataset. After removing the mean, we used the FFT algorithm to estimate the frequency spectrum corresponding to each spatial RF sample in an ROI and then, averaged each component of the spectrum over the ROI. We normalized the average spectrum by setting the maximum of the frequency components to one. The first four features (S1, S2, S3 and S4) were the average values of the normalized spectrum in four quarters of the frequency range. In other words, they summarized the low, mid-low, mid-high, and high frequency components of the signal, respectively. We also fit a regression line to values of the spectrum (versus normalized frequency). The intercept (S5) and the slope (S6) of this line were used as two more features.

Fractal dimension (FD) of RF time series: It has been shown that the fractal dimension of the RF time series computed based on Higuchi's algorithm ${ }^{14}$ contains tissue typing information. ${ }^{5}$ We computed the FD $\left(K_{\max }=16\right)$ of all the time series within an ROI and averaged them to acquire one feature per ROI.

\subsection{SVM classifier}

Support vector machines belong to the family of maximum margin classifiers. Using a kernel function, SVMs map the input data to a higher dimension space where a hyperplane can separate the data into different classes. The process of training a SVM classifier is equivalent to finding this optimal hyperplane in a way that minimizes the error on the training dataset and maximizes the perpendicular distance between the decision boundary and the closest data points in classes. In the two class case, if the training dataset consists of $N$ feature vectors $\left\{x_{1}, \ldots, x_{N}\right\}$ with class labels $y_{i} \in\{1,-1\}$, then the SVM training problem is equivalent to finding $W$ and $b$ such that: ${ }^{15}$

$$
1 / 2 \times W^{T} W+C \sum_{i=1}^{i=N} \xi_{i}
$$


is minimized subject to:

$$
y_{i}\left(W^{T} \phi\left(x_{i}\right)+b\right) \geq 1-\xi_{i}
$$

where $\xi_{i} \geq 0$ are the so-called slack variables that allow for misclassification of noisy and difficult data points, and the parameter $C>0$ controls the trade-off between the slack variable penalty and the margin. ${ }^{15}$ Function $\phi(x)$ maps the data to a higher dimensional space. This new space is defined by its kernel function: $K\left(x_{i}, x_{j}\right)=\phi\left(x_{i}\right)^{T} \cdot \phi\left(x_{j}\right)$. The above problem can be formulated as a quadratic optimization process. The details of the solution and its implementation can be found in. ${ }^{16}$ For the choice of kernel type, we examined linear, sigmoid, polynomial and Gaussian Radial Basis Function (RBF) kernels. We chose the RBF kernel, defined as:

$$
K\left(x_{i}, x_{j}\right)=e^{-\gamma\left\|x_{i}-x_{j}\right\|^{2}}
$$

This was firstly due to the fact that an RBF kernel has only one parameter $(\gamma>0)$ to adjust. This meant that the entire process of adjusting the SVM classifier to a specific problem was reduced to finding the appropriate values for two parameters: $C$ and $\gamma$. Also, we found SVM classifiers based on RBF kernel more accurate in case of our problem. The same two reasons (simplicity of finding the appropriate structure and higher accuracy) were the justification behind choosing the SVM classifier over artificial neural networks (which was used in our previous work ${ }^{3}$ ). Also, the Bayesian classifiers were ruled out due to the non-Gaussian distribution of feature values which complicated the process of defining a priori distributions.

We used the publicly available $\mathrm{C}++$ implementation of the SVM algorithms known as LIBSVM. ${ }^{16}$ For parameter selection $\left(C\right.$ and $\gamma$ ), we exhaustively searched the parameter space $C \in\left\{2^{-10}, 2^{-9}, \ldots, 2^{10}\right\}$ and $\gamma \in\left\{2^{-10}, 2^{-9}, \ldots, 2^{10}\right\}$. For each set of parameters, 10 -fold cross-validation was performed to determine the best SVM parameters separately for each pair of compared phantoms (E1 vs. E2, E3 vs. E4, and $P 1$ vs. $P 2)$.

\subsection{Classification experiments}

We performed pairwise classification experiments with the available phantom types. We had one pair of particle size phantoms ( $P 1$ vs. $P 2)$ and two different pairs of elasticity phantoms (E1 vs. E2) and (E3 vs. $E 4)^{\dagger}$. For each pair, we performed six different experiments. We provide the details of these experiments here. For simplicity, we provide this description for the general phantom pairs $X 1$ and $X 2$. It should be noted that from each phantom type, two physical copies were available ( $X 1, X 1^{\prime}$ for $X 1$ type and $X 2, X 2^{\prime}$ for $X 2$ type).

Experiment 1 - Intra-phantom 1: train on $X 1$ vs. $X 2$, test on $X 1$ vs. $X 2$ : In this experiment, the classifier was trained to separate RF time series ROIs from $X 1$ and $X 2$ phantoms. The classifier was trained on a portion of the data from $X 1$ and $X 2$ phantoms and tested on a different portion of the data from those phantoms. Eight cross sections of RF time series data were available from each phantom. The experiments were performed with a leave-one-out approach. In other words, the classifier was trained on data from seven cross sections of the $X 1$ data vs. seven cross sections of the $X 2$ data and tested on one cross section of $X 1$ vs. $X 2$. The process was repeated for all eight cross sections.

Experiment 2 - Intra-phantom 2: train on $X 1^{\prime}$ vs. $X 2^{\prime}$, test on $X 1^{\prime}$ vs. $X 2^{\prime}$ : This was similar to experiment 1 , however, data from $X 1^{\prime}$ and $X 2^{\prime}$ phantoms was used.

Experiment 3 - Inter-phantom 1: train on $X 1$ vs. $X 2$, test on $X 1^{\prime}$ vs. $X 2^{\prime}$ : In this experiment, the classifier was trained on data from $X 1$ and $X 2$ phantoms and was tested on separating the ROIs of $X 1^{\prime}$ from $X 2^{\prime}$ phantoms. All ROIs from $X 1$ and $X 2$ were used for training and all ROIs from $X 1^{\prime}$ and $X 2^{\prime}$ were used for testing.

Experiment 4 - Inter-phantom 2: train on $X 1^{\prime}$ vs. $X 2^{\prime}$, test on $X 1$ vs. $X 2$ : This was similar to experiment 3 , only the training and testing sets were switched.

${ }^{\dagger} E 1$ and $E 2$ had equal physical properties except for elasticity. The same was true about $E 3$ and $E 4$. Since the experiments were intended to detect the possible elasticity-dependent variations in RF time series, we used $E 1-E 2$ and $E 3-E 4$ as the only pairs used in the pairwise classification experiments on elasticity phantoms. 
Table 4. Classification results on particle size phantoms at $22 \mathrm{fps}$. Analysis of variance (ANOVA) test confirmed that the classification of ROIs in both intra-phantom and inter-phantom experiments was significantly more accurate than the classification in negative control experiment $(p<0.001)$.

\begin{tabular}{c|ccc} 
& Accuracy (std) & Sensitivity & Specificity \\
\hline Intra-phantom (experiments 1 and 2) & $80.5 \%(6.2)$ & $79.8 \%(5.9)$ & $81.1 \%(6.6)$ \\
Inter-phantom (experiments 3 and 4) & $79 \%(3.1)$ & $77.5 \%(3.1)$ & $82.6 \%(3.5)$ \\
Negative control (experiments 5 and 6) & $58 \%(7.1)$ & $62.5 \%(6.1)$ & $60.3 \%(6.9)$ \\
\hline
\end{tabular}

Experiment 5 - Negative control 1: In this experiment, the classifier was trained to separate $X 1$ ROIs from $X 1^{\prime}$ ROIs. Half of the data from each of these phantoms were used for training and the other half was used for testing.

Experiment 6 - Negative control 2: In this experiment, the classifier was trained to separate $X 2$ ROIs from $X 2^{\prime}$ ROIs. Half of the data from each of these phantoms were used for training and the other half was used for testing.

Experiments 1 and 2 were intra-phantom experiments where the training and testing were carried out on the same physical copy of each phantom type. Experiments 3 and 4 were inter-phantom experiments, meaning that training was performed on one copy of X1 and X2 phantoms and testing was performed on the other copies. Experiments 5 and 6 were carried out as negative control tests.

\section{RESULTS}

\subsection{Particle size phantoms}

For particle size phantoms, the results of experiments 1 and 2 when a SVM classifier was used, are presented in Table 4 averaged together as the intra-phantom trials. The average accuracy, sensitivity and specificity (with $5000 \mathrm{E}$ defined as the positive detection), were $80.5 \%, 79.8 \%$ and $81.1 \%$, respectively.

The results of experiments 3 and 4, when a SVM classifier was used, are presented in Table 4 averaged together as the inter-phantom trials. The accuracy slightly dropped to $79 \%$ compared to the intra-phantom experiments. This was expected due to the fact that the training and testing data in inter-phantom trials were from physically different versions of $P 1$ and $P 2$ phantoms.

As Table 4 shows, the average accuracy of the negative control tests where the two classes of ROIs originated from the same phantom types, was $58 \%$. In other words, physically different phantoms which had similar cell sizes were very poorly distinguishable from each other based on the proposed tissue typing approach. Since the phantoms were custom made, small structural differences between the phantoms of the same type were inevitable.

The results showed that RF time series acquired from tissue mimicking phantoms with difference only in the size of cell-mimicking particles were distinguishable with statistically reliable accuracy of $80.5 \%$. The low accuracy of the classification acquired on the negative control experiments involving phantoms with identical cell sizes showed that the method is in fact sensitive to the cell size.

The effect of frame rate: RF time series are generated by repeated scattering of ultrasound beams. The frame rate of the ultrasound machine determines the frequency at which this interaction occurs. We repeated the inter-phantom classification experiments on particle size phantoms with features extracted from RF time series acquired at $45 \mathrm{fps}$ and $15 \mathrm{fps}$. The inter-phantom classification experiments on particle size phantoms with features extracted from RF time series acquired at $15 \mathrm{fps}, 22 \mathrm{fps}$ and $45 \mathrm{fps}$ resulted in accuracies of $74.2 \pm 4.5 \%, 79 \pm 3.1 \%$, and $82.2 \pm 3.2 \%$, respectively. 
Table 5. Classification results on elasticity phantoms.

\begin{tabular}{c|ccc} 
& Accuracy (STD) & $\begin{array}{c}\text { Sensitivity } \\
E 1 \text { versus } E 2\end{array}$ & Specificity \\
\hline Intra-phantom & $75.9 \%(14.9)$ & $78.0 \%(17.2)$ & $73.8 \%(19.2)$ \\
Inter-phantom & $68.9 \%(5.9)$ & $76.5 \%(11.7)$ & $61.3 \%(12.9)$ \\
Negative control & $64.5 \%(8.8)$ & $66 \%(10.1)$ & $62.3 \%(9.3)$ \\
\hline & \multicolumn{3}{|c}{$E 3$ versus $E 4$} \\
\hline Intra-phantom & $92.2 \%(14.9)$ & $89.8 \%(15.8)$ & $94.4 \%(15.7)$ \\
Inter-phantom & $74.9 \%(13.9)$ & $74.4 \%(22.2)$ & $75.5 \%(10.8)$ \\
Negative control & $68.3 \%(18.1)$ & $70.4 \%(15.4)$ & $66.5 \%(11.3)$ \\
\hline
\end{tabular}

\subsection{Elasticity phantoms}

Inter-phantom and intra-phantom classification trials were performed on $E 1-E 2$ and $E 3-E 4$ phantom pairs, separately. Two versions of each type were available $\left(E 1, E 1^{\prime}, E 2, E 2^{\prime}, E 3, E 3^{\prime}, E 4, E 4^{\prime}\right)$ and experiments 1-6 were separately performed for $E 1$ vs. $E 2$ and $E 3$ vs. E4. The results are presented in Table 5 . In case of $E 1$ vs. E2 classification, the accuracy was $75.6 \%$ in the intra-phantom experiments which dropped to $68.9 \%$ in inter-phantom experiment. It is notable that the classification results were low in comparison to the previous experiments, and were also inconsistent over different trials (large STDs presented in Table 5).

For E3 vs. E4 classification, the intra-phantom trials resulted in a high average accuracy (second half of Table 5). However, a sharp reduction of the accuracy in the inter-phantom classification was witnessed (74.9\%). Also, for both cases, the classification results over different trials were very inconsistent (with standard deviations up to $22 \%$ ).

The difference between the Young's modulus of $E 1$ and $E 2$ phantoms was larger than the difference between the Young's modulus of $E 3$ and $E 4$ phantoms (see Figure 3). Contrary to this fact, it appeared that the classification method was more successful in case of E3 vs. E4 problem. More importantly, the negative control classification experiments resulted in relatively high accuracies $(64.5 \%$ and $68.3 \%$ for $E 1-E 2$ and E3-E4 experiments, respectively. See Table 5). This further supports the notion of inconsistent results in the case of elasticity phantoms.

\section{DISCUSSIONS}

Our studies reported in this paper and elsewhere ${ }^{17-19}$ show that the RF time series are strongly affected by the tissue microstructure. Although the electronic noise of the ultrasound system can also affect the variations of RF time series, our studies have shown that the correlation of the RF time series features with the tissue type is strong and can be used for tissue typing. The evidence was obtained in an ex-vivo clinical study that involved 35 patients who underwent the prostatectomy surgery over a period of one year. We were able to detect the prostate cancer tumors and acquire an area under ROC curve of 0.87 in leave-one-out classification experiments. ${ }^{18}$ In other words, for detecting the tumors in the data acquired from a specific patient, the classifier was trained on the data acquired only from the other patients. This result was validated based on the whole-mount histopathologic analysis of the prostate specimens. In these studies, the seven tissue typing features presented in the current paper were used along with SVM for classification of regions of interest of size $3.5 \mathrm{~mm}^{2}$. We conclude that despite the effects of the probe-related variations, the sensitivity of the RF time series to the tissue-type dependent variations is strong and a supervised classifier such as SVM or neural networks can be successfully trained using the proposed features for tissue typing.

Our methodology to demonstrate the utility of RF time series for tissue typing has been experimental. Nevertheless, a potential explanation for the existence of the tissue typing information in RF time series is presented here. Since ultrasound causes a mechanical force in the direction of wave propagation, the existence of the tissue-type dependent information in the RF time series could be attributed to the microstructural vibrations under the low frequency mechanical excitation caused by the sequence of applied ultrasound beams. 
There are published measurements on the magnitude of mechanical vibrations of scattering particles under the emission of ultrasound. ${ }^{20}$ In live tissue, physiological movements caused by spontaneous cell motions ${ }^{21}$ and capillary level blood circulation (both of which depend on the function of the tissue) further pronounce variations in the sequence of $\mathrm{RF}$ echoes.

We have shown that an increase in the level of energy delivered by ultrasound to tissue, increases the tissue typing performance of features extracted from the RF time series: ${ }^{18}$ Using a Sonix RP ultrasound machine and the BPSL9-5/55/10 trans-rectal probe operating at its highest power settings, an area under the receiver operating characteristic (ROC) curve of 0.87 was achieved in ex-vivo studies involving 35 patients. A $2 d B$ reduction in the output acoustic power resulted in a drop of the area under the ROC curve from 0.87 to 0.84 $(p<0.01)$. A $6 d B$ reduction in the acoustic power output resulted in a more significant drop of the area under ROC curve to $0.76(p<0.001)$. According to the results of this study, the tissue-dependent information in RF time series has a direct relationship with the amount of energy applied to the tissue. This supports our hypothesis suggesting that the tissue typing information in RF time series originate from microstructural vibrations in biological tissue caused by the acoustic force from ultrasound beams. The differences between $\mathrm{RF}$ time series received from different tissue types fade at lower levels of energy which supports the idea of a mechanical mechanism as the possible cause of the tissue type related variations in echoes.

\section{CONCLUSION AND FUTURE WORK}

We have shown that the sequence of echoes received from tissue, while the probe and the tissue are fixed in position, bear tissue-type dependent variations. ${ }^{5,18}$ This sequence of echoes is called RF time series. In this paper, we reported phantom studies aimed at identification of physical properties of tissue that affect the ultrasound RF time series. Our analysis was based on seven features extracted from time series of RF ultrasound echoes used along with SVM classification. The results showed that RF time series acquired from tissue mimicking phantoms with difference only in the size of cell-mimicking particles are distinguishable with statistically reliable accuracy of $82.2 \%$ (at the frame rate of $45 \mathrm{fps}$ ). The classification accuracy meaningfully increased with increase in the frame rate. We also studied the tissue typing capabilities of the RF time series on phantoms with differences in elastic properties. Classification of elasticity phantoms show more variability. We conclude that the features extracted from time series of RF signals are strongly affected by the microstructural properties of the phantoms. This was confirmed based on the high accuracy and consistency of the results acquired on particle size phantoms. On the other hand, the low accuracy of classification experiments on elastography phantoms show that our proposed features are not highly sensitive to the macroscopic elasticity of the tissue.

Previously, we have shown that RF time series provide a reliable source of tissue typing features for ex-vivo detection of prostate cancer. ${ }^{4}$ The results of this study confirm that the evident differences in microstructure of cancerous versus normal tissue could play a role in the success of the proposed tissue typing method. The next planned stage of our study is to acquire ultrasound RF time series data from patients undergoing prostate biopsy to confirm the in-vivo utility and sensitivity of the proposed method.

\section{ACKNOWLEDGMENTS}

The authors gratefully acknowledge the assistance of Dr. Jonathan Boisvert (Queen's University) in the phantom building process. This research was funded by the Natural Sciences and Engineering Research Council (NSERC) of Canada and the Institute of Robotics and Intelligent Systems (IRIS).

\section{REFERENCES}

[1] Lizzi, F. L., Feleppa, E. J., Alam, S. K., and Deng, X. C., "Ultrasonic spectrum analysis for tissue evaluation," Pattern Recognition Letters 24, 637-658 (2003).

[2] Jemal, A., Siegel, R., Ward, E., Murray, T., Xu, J., and Thun, M., "Cancer statistics, 2007," CA: $A$ Cancer Journal for Clinicians 57(1), 43-66 (2007). 
[3] Moradi, M., Mousavi, P., Siemens, D. R., Sauerbrei, E. E., Isotalo, P., Boag, A., and Abolmaesumi, P., "Discrete Fourier analysis of ultrasound RF time series for detection of prostate cancer," in [Proceedings of IEEE Engineering in Medicine and Biology Conference], 1339-1342 (2007).

[4] Moradi, M., Mousavi, P., Siemens, D. R., Sauerbrei, E. E., Boag, A., and Abolmaesumi, P., "Ultrasound RF time series for detection of prostate cancer: Feature selection and frame rate analysis," in [Proceedings of IEEE Ultrasonics Symposium], 2493-2496 (2007).

[5] Moradi, M., Mousavi, P., and Abolmaesumi, P., "Tissue characterization using fractal dimension of high frequency ultrasound RF time series," in [Proceedings of Medical Image Computing and Computer-Aided Interventions (MICCAI)], Ayache, N., Ourselin, S., and Maeder, A., eds., LNCS 4792, 900-908 (2007).

[6] Kumar, Abbas, Fausto, and Mitchell, [Basic Pathology], Saunders, Elsevier, PA, USA, 8 ed. (2007).

[7] Kolios, M. C. and Baddour, R. E., "Investigating the effect of cell size on the backscatter from suspensions of varying volume fractions," in [Proceedings of IEEE Ultrasonics Symposium], 637-640 (2006).

[8] Ophir, J., Cespedes, I., Ponnekanti, H., Yazdi, Y., and Li, X., "Elastography: a method for imaging the elasticity in biological tissues," Ultrasonic Imaging 13(2), 111-134 (1991).

[9] Zhang, X., Zeraati, M., Kinnick, R. R., Greenleaf, J. F., and Fatemi, M., "Vibration mode imaging," IEEE Transactions on Medical Imaging 26(6), 843-852 (2007).

[10] Madsen, E. L., Hobson, M. A., Shi, H., Varghese, T., and Frank, G. R., "Tissue-mimicking agar/gelatin materials for use in heterogeneous elastography phantoms," Physics in Medicine and Biology 50(23), 5597-5618 (2005).

[11] Bigelow, T. A. and Jr, W. D. O., "Scatterer size estimation in pulse-echo ultrasound using focused sources: calibration measurements and phantom experiments," Journal of the Acoustical Society of America 116(1), 594-602 (2004).

[12] Erkamp, R. Q., Skovoroda, A. R., Emelianov, S. Y., and O'Donnell, M., "Measuring the nonlinear elastic properties of tissue-like phantoms," IEEE Transactions on Ultrasonics, Ferroelectrics, and Frequency Control 51(4), 410-419 (2004).

[13] Hedrick, W. R., Hykes, D. L., and Starchman, D. E., [Ultrasound Physics and Instrumentation], Elsevier Mosby, St. Louis, MO (2005).

[14] Higuchi, T., "Approach to an irregular time series on the basis of the fractal theory," Physica D 31(2), 277-283 (1988).

[15] Bishop, C. M., [Pattern Recognition and Machine Learning], Springer Science, New York (2006).

[16] Fan, R. E., Chen, P. H., and Lin, C. J., "Working set selection using the second order information for training svm," Machine Learning Research 6, 1889-1918 (2005).

[17] Moradi, M., A New Paradigm for Ultrasound-Based Tissue Typing in Prostate Cancer, PhD thesis, Queen's University, Kingston, ON, Canada (2008).

[18] Moradi, M., Abolmaesumi, P., Siemens, D. R., Sauerbrei, E. E., Boag, A., and Mousavi, P., "Augmenting detection of prostate cancer in transrectal ultrasound images using SVM and RF time series," IEEE Transactions on Biomedical Engineering. In Press (2009).

[19] Moradi, M., Mousavi, P., Isotalo, P. A., Siemens, D. R., Sauerbrei, E. E., and Abolmaesumi, P., "A new approach to analysis of RF ultrasound echo signals for tissue characterization: results of animal studies," in [SPIE Medical Imaging], Emelianov, S. Y. and McAleavey, S. A., eds., 6513, 65130P1-65130P10 (2007).

[20] Konofagou, E. E. and Hynynen, K., "Localized harmonic motion imaging: Theory, simulations and experiments," Ultrasound in Medicine and Biology 28(10), 1405-1413 (2003).

[21] Takagi, H., Sato, M. J., Yanagida, T., and Ueda, M., "Functional analysis of spontaneous cell movement under different physiological conditions," PLOS ONE 3(7), 1-7 (2008). 